\title{
Genome Size Is a Strong Predictor of Root Meristem Growth Rate
}

\author{
Adam Gruner, Nathan Hoverter, Tylia Smith, and Charles A. Knight \\ Department of Biological Sciences, California Polytechnic State University, San Luis Obispo, CA 93407, USA \\ Correspondence should be addressed to Charles A. Knight, knight@calpoly.edu
}

Received 23 December 2009; Accepted 5 March 2010

Academic Editor: Johann Greilhuber

Copyright (C) 2010 Adam Gruner et al. This is an open access article distributed under the Creative Commons Attribution License, which permits unrestricted use, distribution, and reproduction in any medium, provided the original work is properly cited.

\begin{abstract}
Variation in genome size (GS) has been linked to several facets of the plant phenotype. Recently it was shown that GS is significantly correlated with cell size and the duration of the cell cycle. Here we test the hypothesis that GS might also be a predictor of apical root meristem growth rate (RMGR). We studied eight species of eudicots with varying GS using time-lapse microscopic image analysis. A significant negative exponential relationship was observed between GS and RMGR. Our results show significantly decreased RMGR for large genome species. This relationship represents a significant consequence of GS expansion in plants and may partly explain why genome sizes tend to be small in eudicots. Interestingly, parasitic plants, which do not rely on root growth as much, often have large genomes.
\end{abstract}

\section{Introduction}

Genome size (GS) varies by three orders of magnitude in plants [1, 2]. Correlations between GS and several facets of the phenotype and life history of plants have been documented $[3,4]$. The strongest relationships are found at the cellular level. For example, GS is positively correlated with cell size $[5,6]$. In addition, Francis et al. [7] found a consistent positive relationship between GS and the duration of the cell cycle, which confirmed earlier observations by Bennett $[8,9]$. Here we test the hypothesis that GS may be a predictor of meristem growth. We chose to study root meristem because it is relatively easy to model and quantify along a single primary axis. Others have observed correlations between the relative growth rate and genome size $[4,10,11]$, but none have looked specifically at growth dynamics (kinematics) in the root meristem.

Apical root meristem growth rate (RMGR) can be modeled with the following equation:

$$
\mathrm{RMGR}=N^{(R \Delta T)}\left(\frac{\Delta L}{\Delta T}\right),
$$

where RMGR is a function of the number of initial cells $(N)$, the division rate of these cells $(R)$, the time it takes for a point to pass through the elongation zone $(\Delta T)$, and the change in length of a cell $(\Delta L)$ over the change in time $(\Delta T)$. All of the independent variables in (1) can be measured using timelapse microscopic image analysis $[12,13]$. We measured root kinematics for seven replicates of eight eudicot species with varying GS to test the hypothesis that GS is a predictor of RMGR (Table 1). Assuming that the number of initial cells remains fairly constant between species, variation in RMGR could be caused by variation in the rates of cell division $(R)$ and/or the elongation rate of these cells $(\Delta L / \Delta T)$. Variation in cell division rate would lead to an exponential relationship between genome size and RMGR; variation in cell elongation rate would lead to a linear relationship. Therefore, we tested both exponential and linear models.

\section{Methods}

2.1. Seed Preparation and Incubation. We measured root growth velocity for eight species (Table 1). All seeds were obtained from Botanical Interests, Inc. (http://www.botanicalinterests.com/). Seeds were sterilized in $6 \% \mathrm{HCl}$ bleach then rinsed in deionized water. Two seeds of like species were placed on a $100 \times 15 \mathrm{~mm}$ sterile agar plate. Our agar contained $0.6 \%(\mathrm{~m} / \mathrm{v})$ Phytagel (SigmaAldrich, USA) medium containing $0.4 \%(\mathrm{v} / \mathrm{v})$ nutrient solution (7-9-5 Liquid Grow, Dyna-Gro, San Francisco, CA). The medium was buffered to a $\mathrm{pH}$ of 5.8 with $0.05 \%$ $(\mathrm{m} / \mathrm{v})$ MES. Plates were wrapped with parafilm which 
TABle 1: Genome size, elongation zone length, and root tip velocity for eight species of eudicots. Data are the means \pm SE of at least seven replicates.

\begin{tabular}{lccc}
\hline Species & $\begin{array}{c}\text { Genome size } \\
(1 \mathrm{C} \text {-value - Gbp })\end{array}$ & $\begin{array}{c}\text { Elongation zone length } \\
(\mu \mathrm{m})\end{array}$ & $\begin{array}{c}\text { RMGR } \\
\left.(\mu \mathrm{m} \mathrm{min})^{-1}\right)\end{array}$ \\
\hline Citrullus vulgaris & 0.45 & $2010 \pm 316$ & $6.29 \pm 1.01$ \\
Cucurbita pepo & 0.55 & $2426 \pm 354$ & $7.06 \pm 0.99$ \\
Brassica rapa & 0.80 & $786 \pm 41$ & $4.96 \pm 0.42$ \\
Lycopersicon esculentum & 1.01 & $953 \pm 48$ & $5.37 \pm 0.41$ \\
Cosmos bipinnatus & 1.52 & $108 \pm 101$ & $4.60 \pm 0.79$ \\
Cosmos sulphureus & 2.94 & $937 \pm 79$ & $4.02 \pm 0.42$ \\
Capsicum annuum & 3.92 & $1196 \pm 120$ & $2.89 \pm 0.26$ \\
Capsicum frutescens & 5.88 & $871 \pm 63$ & $2.17 \pm 0.19$ \\
\hline
\end{tabular}

was then perforated to allow gas exchange. Seeds were incubated inside a growth chamber (CMP4030 incubator, Conviron, Winnipeg, Canada) at a $45^{\circ}$ angle under 24 -hour full light at $25^{\circ} \mathrm{C}$. Genome sizes for the species we studied were obtained from the plant DNA C-values database (http://data.kew.org/cvalues/).

2.2. Determining Root Kinematics. We collected microscopic images of roots using a Nikon compound microscope (Nikon Optiphot-2, Nikon, Tokyo, Japan) fitted with a 10/0.30 objective (Nikon Plan, Nikon, Tokyo, Japan) and a digital camera (QICAM-IR, Q-Imaging, Surrey, Canada). Stacks of nine images were taken along the length of the root using a constant interval of 20 to 120 seconds between frames. Each root was assayed between 2 and 14 days after germination. Each plate with a viable root was removed from the incubator and positioned with the root flush to the superior side. Graphite particles were sprinkled along the length of the root using a small paintbrush. These particles were used to produce a background image for each image stack to give a reference for splicing the image stacks together.

We used RootflowRT [13], developed by Tobias Baskin and colleagues, to determine root kinematics from these image stacks (version 2.8, University of Massachusetts-Amherst, available at http://www.bio.umass.edu/biology/baskin/). RootflowRT uses dynamic high contrast image stacks and static background images to create a linear velocity profile of a root. The program determines velocity at various positions by tracking pixels moving along a mapped pathway. The image stacks are first converted to.tif format using IrfanView (http://www.irfanview.com/). The centerline of the fifth image in each stack is then visualized using ImageJ (http://rsbweb.nih.gov/ij/) and points along the line are plotted into the input file. The quiescent center is also entered into the input file and used as the point of origin for velocity determination. The output plot of velocity versus position is a sigmoidal curve representing the three regions of meristematic development. The curve plateaus in the zone of maturation. In this region the root shows little longitudinal growth but begins to grow radially and root hairs and lateral roots appear. For the purposes of this study we focused on the maximum growth rate (the velocity of the quiescent center relative to the zone of maturation).

\section{Results and Discussion}

There was a significant negative relationship between genome size and RMGR (Figure 1(a)). The trend was remarkably linear across all species. Phylogenetically independent species pairs (Cosmos and Capsicum) with contrasting genome sizes also showed the same trend: the species with the larger genome size had a significantly slower root growth velocity (Table 1 ) and a longer elongation zone. The exponential $\left(F_{\mathrm{df}=6}=\right.$ 97.32, $P \leq .0001, r^{2}=0.94$; Figure $1(\mathrm{a}))$ and linear $\left(F_{\mathrm{df}=6}=36.64, P \leq .001\right.$, and $\left.r^{2}=0.86\right)$ models were both significant; however, the exponential model was stronger. This result suggests that cell division rate has a larger effect on root meristem growth rate than does variation in cell expansion rate. However, these two models (linear versus exponential) are not mutually exclusive. Decreased cell division rates and elongation rates may both be responsible for decreased RMGR in species with a large GS. If the linear model is correct, the RMGR of eudicots would approach zero at a GS of less than $10 \mathrm{bbp}$. But many eudicot species are known to have a GS larger than $10 \mathrm{bbp}$ (Figure 1(b)), further supporting the exponential model where changes in cell division rate are the primary driver of changes in RMGR.

Continuous root growth is necessary for plants. Roots must expand into new areas as nutrients are depleted in the nearby soil [14]. As a root grows, it acidifies the environment to facilitate the cotransport of nutrients into the root [15]. This acidification also acts to trigger expansin activity in cell walls, which breaks cross bridges between cellulose microfibrils, leading to cell expansion [16]. Nutrients are absorbed primarily in this area of loosened cell walls in the elongation zone. Therefore, reduction in root growth rate may represent a significant consequence of genome size expansion in plants. A slower RMGR could limit a species to nutrient-rich soils, acidic soils, or require it to find an alternative means of nutrient absorption, such as parasitism [17] or mycorrhizal symbiosis [18]. Currently, 


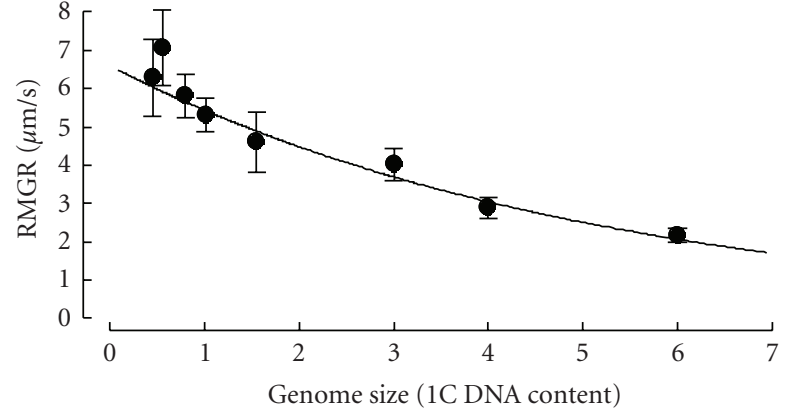

(a)

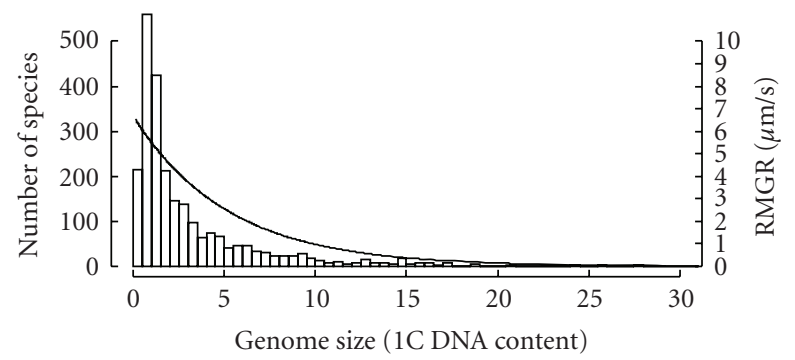

(b)

Figure 1: (a) The relationship between genome size (GS) reported in gigabasepairs, and root meristem growth rate (RMGR) for eight eudicot species with the best fit exponential regression line. Error bars are the standard error of the mean for seven replicates for each species. (b) Frequency distribution of genome sizes for eudicots with the best fit line from (a) overlaid.

there are 2,459 species of eudicots that have a known GS. The number of representative species approaches zero at a GS of 25-30 bbp (Figure 1(b)). This is the same GS at which the RMGR approaches zero with our exponential prediction (Figure 1(a)). The only eudicot genus containing species with GS above $30 \mathrm{bbp}$, Viscum, contains parasitic species (mistletoes) which do not use roots to absorb nutrients [17] and can therefore bypass this limitation.

Large genome species may require additional time for DNA synthesis, thus leading to increased cell-cycle duration $[5,6]$. However, we hypothesize that it may also involve decreased efficiency of signal transduction for gene expression. For example, a larger GS may increase the time required for a transcription factor to find the proper regulatory element or increase mismatch frequency. This would make gene expression less responsive to endogenous signals. Genes involved in cell-cycle regulation are just one example of an expression system that could be slowed with increasing GS, perhaps resulting in the patterns we observed here. This hypothesis should be tested in a comparative framework. In addition, RMGR should be measured in monocots and gymnosperms, which contain species with significantly larger GS than eudicots.
GS is a nongenic factor that has phenotypic consequences. The patterns we describe here are perhaps the strongest and most significant phenotypic correlations with GS described to date. Further analyses should examine these trends in other plant groups.

\section{Acknowledgment}

This project was supported by the Office of Graduate Studies and the Biological Sciences Department at California Polytechnic State University, San Luis Obispo,USA.

\section{References}

[1] T. R. Gregory, The Evolution of the Genome, Elsevier Academic Press, Burlington, Vt, USA, 2006.

[2] M. D. Bennett and I. J. Leitch, "Plant DNA C-values database," release 4.0, October 2005, http://www.kew.org/genomesize/ homepage.

[3] C. A. Knight and J. M. Beaulieu, "Genome size scaling through phenotype space," Annals of Botany, vol. 101, no. 6, pp. 759766, 2008.

[4] C. A. Knight, N. A. Molinari, and D. A. Petrov, "The large genome constraint hypothesis: evolution, ecology and phenotype," Annals of Botany, vol. 95, no. 1, pp. 177-190, 2005.

[5] J. M. Beaulieu, I. J. Leitch, S. Patel, A. Pendharkar, and C. A. Knight, "Genome size is a strong predictor of cell size and stomatal density in angiosperms," New Phytologist, vol. 179, no. 4, pp. 975-986, 2008.

[6] J. A. Connolly, M. J. Oliver, J. M. Beaulieu, C. A. Knight, L. Tomanek, and M. A. Moline, "Correlated evolution of genome size and cell volume in diatoms (Bacillariophyceae)," Journal of Phycology, vol. 44, no. 1, pp. 124-131, 2008.

[7] D. Francis, M. S. Davies, and P. W. Barlow, "A strong nucleotypic effect on the cell cycle regardless of ploidy level," Annals of Botany, vol. 101, no. 6, pp. 747-757, 2008.

[8] M. D. Bennett, "The duration of meiosis," Proceedings of the Royal Society of London. Series B, vol. 178, pp. 259-275, 1971.

[9] M. D. Bennett, "The time and duration of meiosis," Philosophical Transactions of the Royal Society of London. Series B, vol. 277, no. 955, pp. 201-226, 1977.

[10] M. D. Bennett, "Nuclear DNA content and minimum generation time in herbaceous plants," Proceedings of the Royal Society of London. Series B, vol. 181, no. 63, pp. 109-135, 1972.

[11] M. A. Mowforth and J. P. Grime, "Intra-population variation in nuclear DNA amount, cell size and growth rate in Poa annua L.," Functional Ecology, vol. 3, no. 3, pp. 289-295, 1989.

[12] G. T. S. Beemster and T. I. Baskin, "Analysis of cell division and elongation underlying the developmental acceleration of root growth in Arabidopsis thaliana," Plant Physiology, vol. 116, no. 4, pp. 1515-1526, 1998.

[13] C. M. Van Der Weele, H. S. Jiang, K. K. Palaniappan, V. B. Ivanov, K. Palaniappan, and T. I. Baskin, "A new algorithm for computational image analysis of deformable motion at high spatial and temporal resolution applied to root growth. Roughly uniform elongation in the meristem and also, after an abrupt acceleration, in the elongation zone," Plant Physiology, vol. 132, no. 3, pp. 1138-1148, 2003.

[14] X. Li, E. George, and H. Marschner, "Phosphorus depletion 
and $\mathrm{pH}$ decrease at the root-soil and hyphae-soil interfaces of VA mycorrhizal white clover fertilized with ammonium," New Phytologist, vol. 119, no. 3, pp. 397-404, 1991.

[15] P. Imas, B. Bar-Yosef, U. Kafkafi, and R. Ganmore-Neumann, "Release of carboxylic anions and protons by tomato roots in response to ammonium nitrate ratio and $\mathrm{pH}$ in nutrient solution," Plant and Soil, vol. 191, no. 1, pp. 27-34, 1997.

[16] B. M. Link and D. J. Cosgrove, "Acid-growth response and $\alpha$ expansins in suspension cultures of bright yellow 2 tobacco," Plant Physiology, vol. 118, no. 3, pp. 907-916, 1998.

[17] P. Escher and H. Rennenberg, "Influx of double labelled glutamine into mistletoes (Viscum album) from the xylem sap of its host (Abies alba)," Plant Physiology \& Biochemistry, vol. 44, no. 11-12, pp. 880-884, 2006.

[18] H. Marschner and B. Dell, "Nutrient uptake in mycorrhizal symbiosis," Plant and Soil, vol. 159, no. 1, pp. 89-102, 1994. 

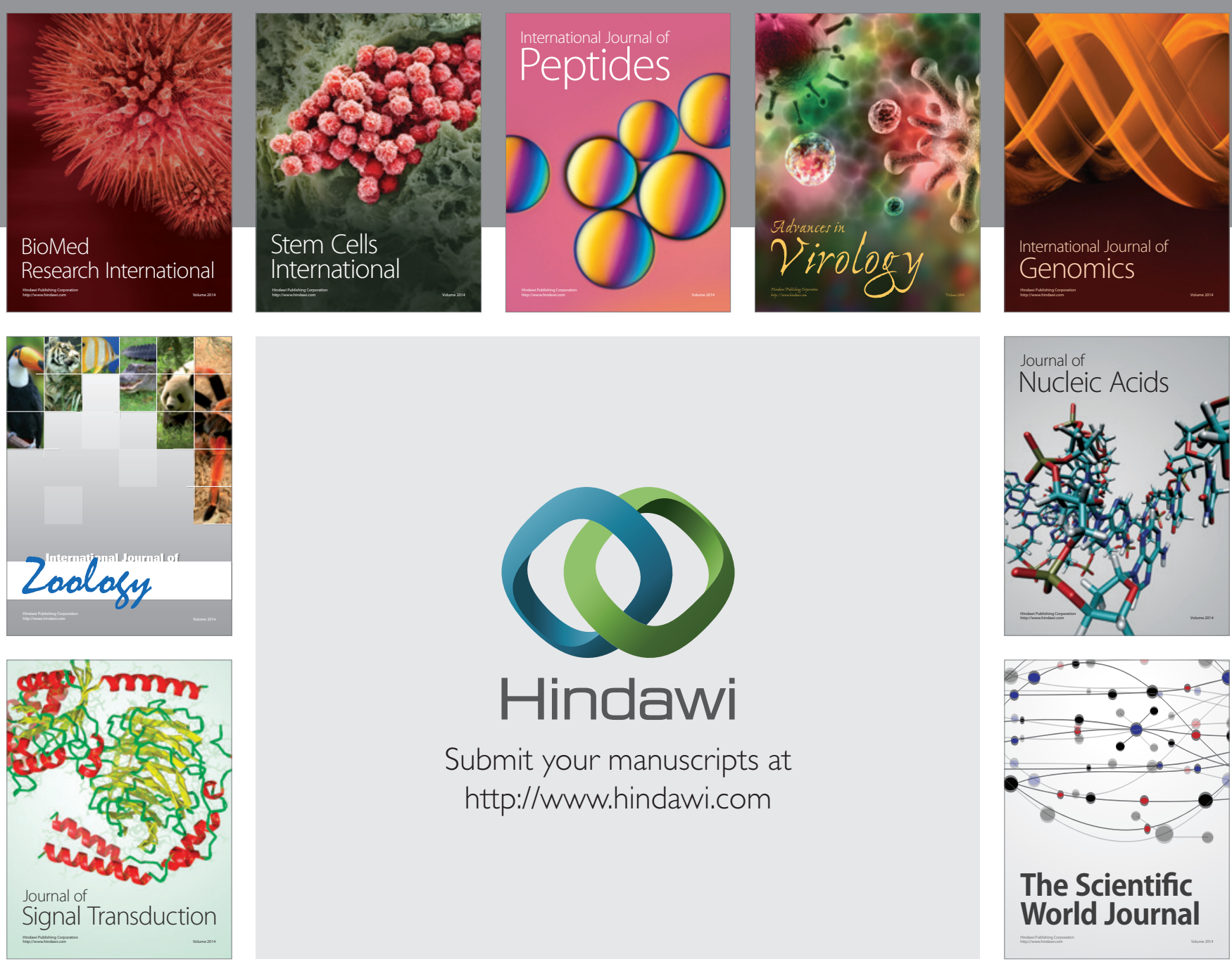

Submit your manuscripts at

http://www.hindawi.com
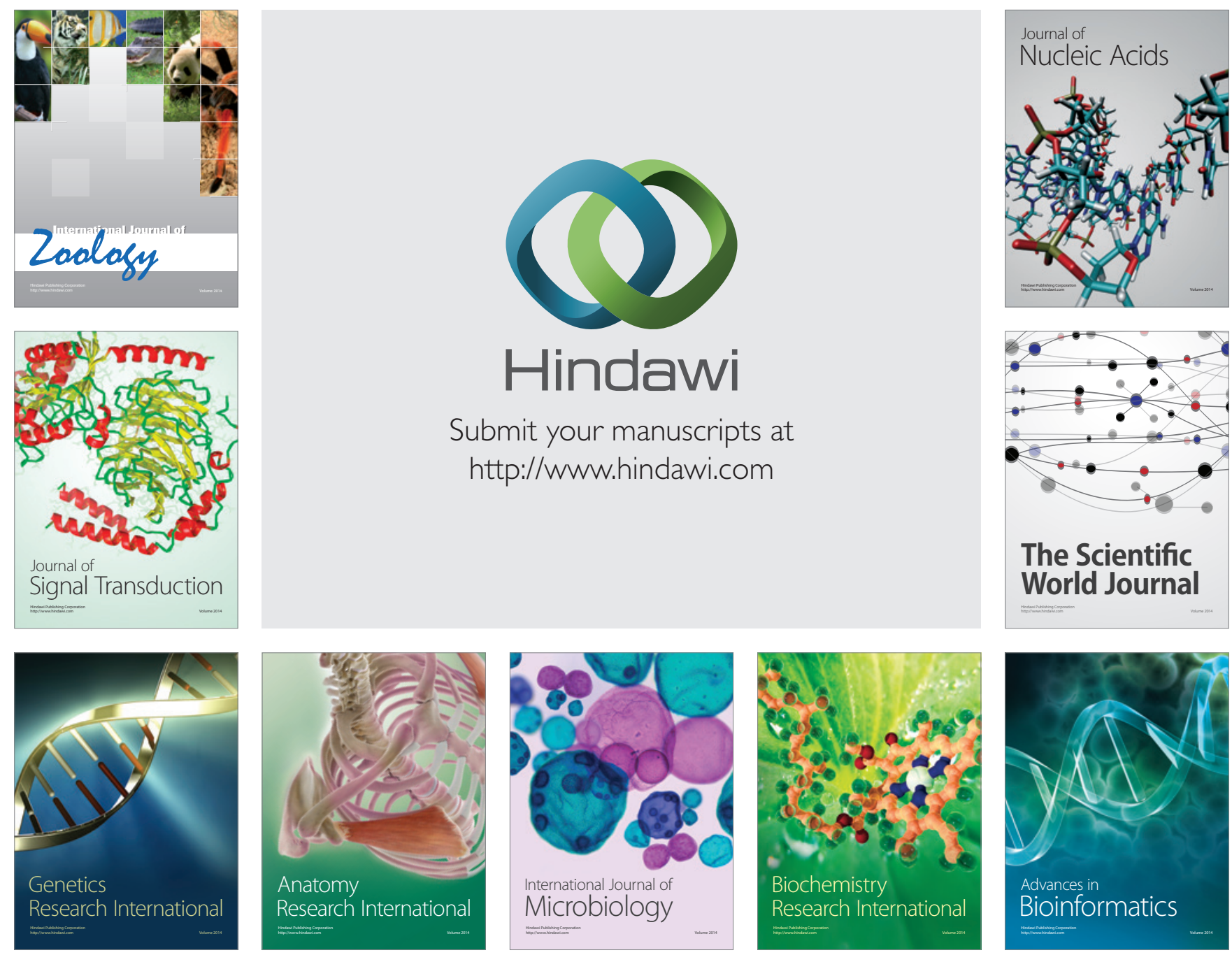

The Scientific World Journal
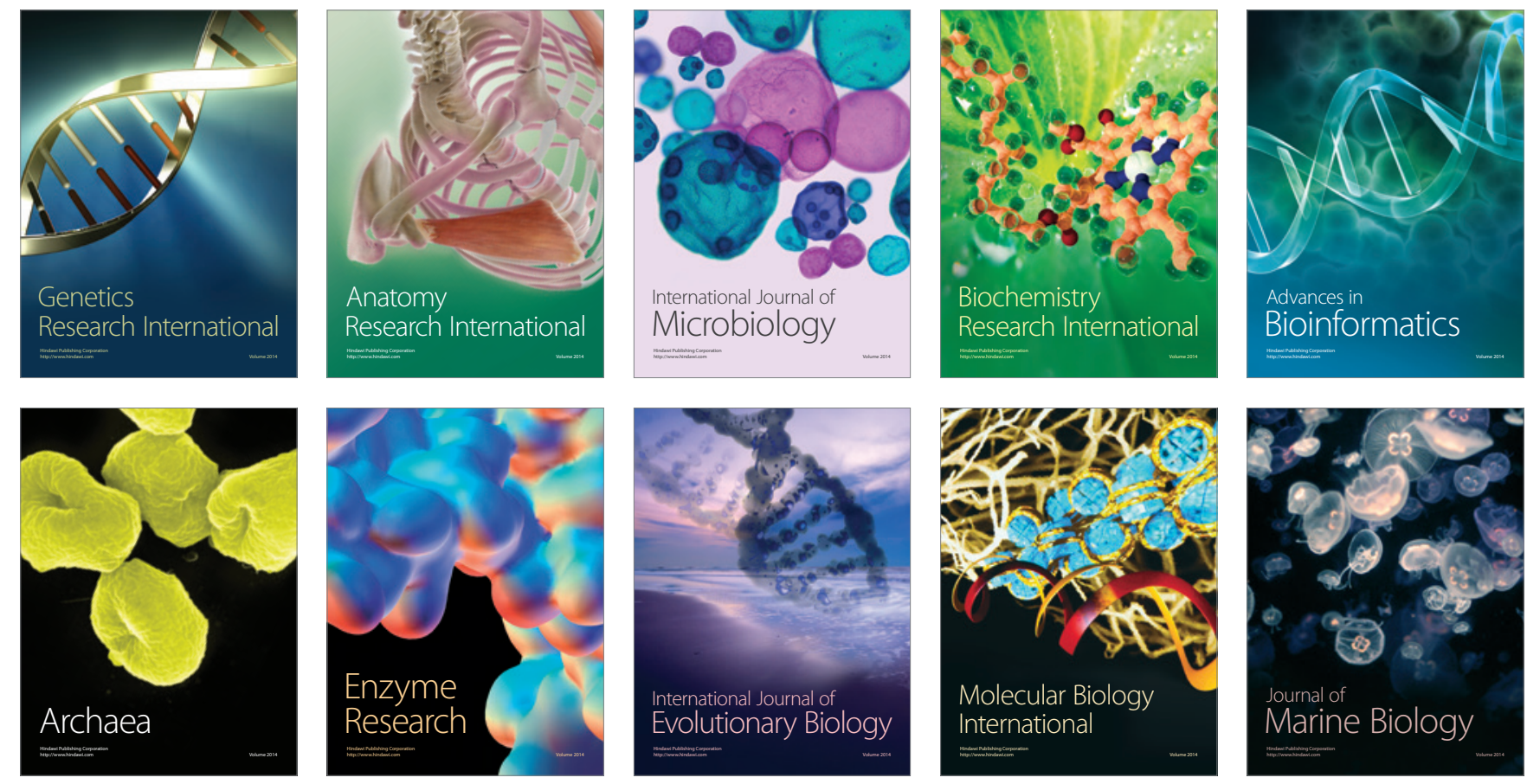